\title{
List of Editors and Contributors
}

Cong CAO is a Senior Research Associate with the Neil D. Levin Graduate Institute of International Relations and Commerce at the State University of New York where he also coordinates Levin's Global Talent Index Project. He received his PhD in sociology from Columbia University and has worked at the University of Oregon and the East Asian Institute at the National University of Singapore. $\mathrm{He}$ is interested in the social studies of science and technology with a focus on China. He is the author of China's Scientific Elite (London and New York: RoutledgeCurzon, 2004), a study of Chinese scientists holding elite membership in the Chinese Academy of Sciences, and Talent and China's Technological Edge with Denis Fred Simon (Cambridge and New York: Cambridge University Press, forthcoming).

Michael HENG Siam-Heng is a Senior Research Fellow at the East Asian Institute, National University of Singapore. He has held academic appointments in Australia, China, Malaysia, The Netherlands and Singapore. He holds a BSc (Hons) in physics, Diploma in Education, MSc in computer studies and $\mathrm{PhD}$ in information systems (Vrije Universiteit, Amsterdam). He was a winner of the Emerald Literati 


\section{List of Editors and Contributors}

Network Award for Excellence in 2006 for his paper on supply chain management. He is the Asia-Pacific Editor of the International Journal of Electronic Customer Relationship Management, an Associate Editor of the Journal of Electronic Commerce Research and an editor of the International Journal of Value Chain Management. He co-edited Supply Chain Management: Issues in the New Era of Collaboration and Competition (Hershey, PA: Idea Group Publishers, 2007). His research interests cover globalisation, electronic business, information systems strategy and organisational change.

Albert Guangzhou HU is Associate Professor of Economics at the National University of Singapore. He received his BA in international finance from Nankai University and PhD in international economics from Brandeis University. His research interests include the economics of technological change, international economics, industrial organisation, and in particular, the Chinese economy. His research has appeared in such journals as China Economic Review, Economic Development and Cultural Change, International Journal of Industrial Organisation, Journal of Comparative Economics, Research Policy and Review of Economics and Statistics. He has also been a short-term consultant for the World Bank.

Johan LAGERKVIST is a Senior Research Fellow with The Swedish Institute of International Affairs (SIIA). He received his $\mathrm{PhD}$ in Chinese from Lund University. His dissertation, China and the Internet: Unlocking and Containing the Public Sphere, was published by Lund University Press in 2006. He has published articles and book chapters in several international scientific journals and research anthologies, as well as served as guest editor for two theme issues of Contemporary Chinese Thought. He is a board member of the Nordic Association for China Studies. He is currently working on a research project financed by the Swedish Research Council on issues of law implementation in China. In another project supported by SIIA, he is researching the implications of Chinese nationalism on the peaceful development policy of China. 
Ding LU is Professor of Economics at Sophia University, Tokyo. A graduate from China's Fudan University, he obtained his PhD from Northwestern University. Before joining Sophia University, he was at the National University of Singapore and University of Nebraska at Omaha. His research interests include international trade and investment, and regional economic development. He has published several books and dozens of papers in peer-reviewed journals and book chapters. Most of his publications pertain to development issues in Pacific Asia, particularly the Chinese economy. His books include Entrepreneurship in Suppressed Markets: China's Private Sector Experience (New York: Garland, 1994), State Intervention and Business in China: the Role of Preferential Policies (UK: Edward Elgar, 1997), and China's Telecommunications Market: Entering a New Competitive Age (UK: Edward Elgar, 2003).

Yue MA is Professor of Economics at Lingnan University, Hong Kong. He obtained his BSc in Mathematics from Xiamen University and $\mathrm{PhD}$ in Economics and Econometrics from Manchester University. He specialises in the economics of exchange rates and banking, with particular reference to the Mainland Chinese and Hong Kong economies, His publications have appeared in Economic Journal, China Economic Review, European Economic Review, Singapore Economic Review and Journal of International Money and Finance. $\mathrm{He}$ is a member of the editorial board of the Journal of Chinese Economic and Business Studies, UK, and International Trade (Guoji maoyi wenti), Beijing.

Ping LIN is Professor of Economics at Lingnan University, Hong Kong. He received his $\mathrm{PhD}$ from the University of Minnesota. His research interests include industrial economics, foreign direct investment and technology transfer, competition policy, and the theory of banking. His publications have appeared in the Journal of Economic Theory, European Economic Review, Journal of International Economics, Journal of Industrial Economics, Oxford Economic Review and International Journal of Industrial Organization. He has been a 
consultant to the Asian Development Bank, the Government of Hong Kong Special Administrative Region, the Organization for Economic Co-operation and Development and the state Council of the People's Republic of China.

Jon SIGURDSON is Professor in Research Policy, and Director of the East Asia Science and Technology and Culture Programme at the Stockholm School of Economics. He has degrees in engineering and economics (University of Technology and Gothenburg University, respectively). He has had visiting professorships in Japan and Australia and been a Senior Visiting Research Fellow at the East Asian Institute of the National University of Singapore. His most recent publication is Technological Superpower China (Northampton, MA: Edward Elgar, 2006). As a researcher in science policy he has developed special expertise in technology management at national and company level with a focus on areas such as: (1) global structures for research and development and their interaction with national systems for innovation; and (2) structural changes in the electronics and information technologies industries. His present research focus is regional changes and dynamic city developments in China.

Richard P. SUTTMEIER is Professor Emeritus of Political Science at the University of Oregon. He did his BA at Darmouth College in philosophy and his $\mathrm{PhD}$ in political science at Indiana University. His research interests include Chinese science and technology policies, the management of environmental and technological risks in China, high technology development in China, comparison of Chinese and Indian science and technology policies and international perspectives on the ethics of new technologies. He has served as a consultant to the International Development Research Center of Canada, Senior Analyst at the Congressional Office of Technology Assessment, consultant to the World Bank and the United Nations Development Programme, and as the Director of the Beijing Office of the Committee for Scholarly Communication with China. 
Elspeth THOMSON is a Visiting Research Fellow at the East Asian Institute, National University of Singapore, and co-edits EAI's journal, China: An International Journal. She received her PhD in Chinese Economic History from the School of Oriental and African Studies, University of London. Her main research interests are Asian energy and transport. She authored The Chinese Coal Industry: An Economic History (London and New York: RoutledgeCurzon, 2003) and another edited volume, Energy Conservation in East Asia: Towards Greater Energy Security, will also appear in 2008. She has published articles concerning various aspects of Asia's energy sector in The China Quarterly, Pacific and Asian Journal of Energy, Journal of Applied Statistics, China Review, East Asia: An International Quarterly, and Perspectives. Through the 1990s she taught at Simon Fraser University in Vancouver and Lingnan University in Hong Kong.

YAO Xiangkui is currently a $\mathrm{PhD}$ candidate in Computer and Information Science at the University of Oregon. He received his BA in journalism from the Institute of International Relations in Beijing and his MA in Political Science from the University of Oregon. His research area is requirements engineering and wearable computing. He is a student member of the Association for Computing Machinery.

Jing A. ZHANG is Assistant Professor of Strategic Management at the Faculty of Commerce, Chuhai College of Higher Education Hong Kong. She received her PhD from the University of Wollongong. Zhang's research interests focus on innovation and corporate strategies, firm resources/capabilities and innovation, and strategic management. She has published articles in international journals such as International Journal of Business Studies and RED Management. She is also a keen supporter of the profession through the membership of several professional associations including Asia Academy of Management, and China Association of Science and Technology Indicators. 
This page intentionally left blank 


\section{Index}

Absorption 4, 18, 22, 23, 28, 47, 58,

$$
61,74,163
$$

Academia ix, 8, 20, 23, 33, 54, 63, 87, 90, 94, 145, 182, 194, 200, 202, 207, 211

Aerospace (or aircraft) 10, 12-14, 25, 74, 123, 138

Agglomeration forces ix, xv

Agreement on Trade Related Investment Measures (TRIMS) 69-72

Agreement on Trade-Related Aspects of Intellectual Property Rights (TRIPS) 36, 49, 69, 70

Analog technology 19

Appliances xvi, 145, 146, 167, 180

Applications xii, 4, 10, 24, 25, 33, 43, 47-50, 52-58, 60, 161, 171

Asia $27,81,86,94,100,102,156$, $158,160,161,163,167,174,176$, 181, 184, 185, 202

Asian Financial Crisis (AFC) 67

Assembling 10, 13-16, 180

Assimilation $\quad 4,18,22,23,27,28,85$

Audio 93-95

Automobiles (car) 20, 64, 145, 154

Audio Video Standard (AVS) 86,

$$
\text { 93-95, 105, 106, 112, 113, } 116
$$

Bachelor of Arts (BA degrees) 152, 166

Bank loans xiv, 124, 127, 132, 137, 138
Beijing $3,4,7,8,10,12,18,20-22$, 24-26, 28, 31, 34, 35, 37-40, 63, 72, 80-83, 85, 86, 90, 93, 95-99, 101, 115, 122, 131, 151, 152, 154, 159, 163, 166, 167, 170, 180-182, 191-195, 198-200, 202

Biotechnology xv, 9, 10, 13, 138, 176 Bird headsets 19, 154-156

Blueprints 23

Brain drain $\mathrm{xi}, \mathrm{xv}, 21$

Budget 125, 139, 141

Bureaucracy x, xi, 28, 105, 114, 200, 201

Business participation xi, 33

California 178,185

Cameras 28

Capability $x, x i, 2,3,5,6,16,20,28$, 29, 31, 36, 73, 77, 108, 120, 165, 169, 178, 186-188

Capacity xviii, 17, 27-29, 55, 67, 98, 110, 114, 154, 179, 180, 183

Capital ix, xi, 26, 29, 36, 37, 41, 42, 48, 63, 73, 126, 129, 144, 155, 162, 180, 183, 186

CDMA Code Division Multiple Access 2, 6, 20, 90, 92

Cell phones (see mobile phones) xv, xvi, 90, 108, 155 


\section{Index}

Central planning $\quad \mathrm{x}, \mathrm{xvi}, 33,48$

Central processing units (CPU) 3, 10, 182

Chemicals 147

China National Institute of

Standardisation 85

Chinese Academy of Sciences

(CAS) 20, 23, 24, 89, 94, 105, 145, 147, 148, 151, 182

Chinese Communist Party (CPC) xvii, 193-202, 204, 205

Chips Industry (chips) xvi, 10, 164, 165, 174-176, 178-180, 187

Coastal areas $\mathrm{xv}, \mathrm{xvii}, 144,146,159$

Colleges xv, 9, 149, 150, 152-154, 161, 168, 169, 210, 211

Commission of Science, Technology and Industry for National

Defence 87

Comparative advantage 11, 14-16, 27

Competition xii, 2, 4-6, 8, 9, 11-13,

15-17, 19-21, 26, 28, 33, 47, 53, $55,60,61,63,72-77,79,80,85$, 86, 88-90, 96, 97, 99, 106, 107, 110-116, 119, 131, 140, 157, $170,171,176,178,180,183$, 185, 194, 208, 209

Components xi, 14, 19, 109, 145-147, $156,157,174,176,177,187$, 200, 201

Computer 4, 9, 10, 12-15, 20, 24, 65, 74, 94, 138, 145-148, 150, 158, 163-165, 167-169, 171, 182, 207, 211

Concentration xii, 48, 60-63, 74, 144, $145,150,155,156,177$

Confucian 191, 192, 198-201, 203, 204

Content 14, 70-72, 174, 193, 194, 196, 204

Contract value 39,40

Copyright 36, 69, 93
Cost $\quad x i v, x v, 3,6,10,14,15,17,18$, 27, 70, 72, 76, 93, 112, 113, 126-129, 131-133, 137, 144, 149, 152, 156, 157, 167, 169, $175,179,182,185$

Credit 75, 102, 128, 129, 136, 139 Crowding out 64

Culture ix, xvii, 23, 29, 150, 193, 198, 201, 203-205

Customers 19, 73, 128, 156, 171, 181, 184,203

Dalian xv, 143, 144, 157-171

Dell 21, 97

Demand xvi, xvii, 3, 13, 17, 23, 76, 101, 127, 128, 130, 132, 133, 135, 136, 146, 151, 157, 158, 168, 174, 175, 179, 182, 183, 192-194, 198, 199, 201, 203

Digital xvi, 20, 28, 93, 95, 96, 99-101, 108, 112, 113, 158, 162, 164, $167,171,179,184$

Diversity xi, xvii, 28, 61, 87, 89, 105, 110, 111, 113, 114, 116, 153, 204 DVD players 18

Economic growth $\mathrm{x}, 2,16,119,199$ Economic reforms xii, 36, 201

Economies of scale $10,60,74,128$ Electronics $\mathrm{xv}, 2,3,7,9,10,12,13$, 16, 18, 19, 27, 70, 81, 87, 96, 97, 138, 145, 149, 157, 167, 174-176, 180, 182, 184, 187

Employees 8, 9, 21, 49, 164, 166, 168, 169, 182

Employment (employer) 17, 54, 117, $146,155,176$

Engineering $\quad x v, 11,12,14,21,29,57$, 94, 149, 150, 153, 154, 157, 163, $166,171,185,187$

Engineers 5, 9, 54, 81, 144, 154, 155, $161,166-171,178$ 
Entrepreneurial spirit ix, xi, xv, xvi, xvii, 28, 29, 131, 147, 152, 188, 193, 201, 209

Europe 6, 9, 109, 162, 181, 184

Enhanced Versatile Disk

(EVD) 95-99, 105, 106

External design xii, 49, 52, 53, 55, 60, 77

Fab 179, 180, 181, 184, 185

Factory $x, 182$

Finance ix, xiv, 50, 128, 129, 137, $152,169,194$

Financial resources $\mathrm{x}, 21,23,154$, 185,187

Firms $\quad x, x i v, x v i, x v i i, 1,3,4-7$, 16-19, 21, 24, 25, 27-30, 32, 35, 40, 41, 47, 48, 50, 55, 57, 60, 61, 63, 64, 71-77, 80-82, 91-94, 108, 111, 114, 119, 121, 124, 125, 127-130, 133, 134, 139, 151, 168, 175, 178, 179, 181, 183-188

Foreign direct investment (FDI) xi, xii, 1, 5, 6, 14, 16, 26, 27, 33, 67, 70-74, 77, 145, 147, 157, 163

Fortune 500 club 28

General Agreement on Tariffs and Trade (GATT) 69

General Agreement on Trade in Services (GATS) 69

Generation x, xiv, 2, 4, 5, 45, 104, $124,125,147,180,199$

Germany $6,32,67,68,83,121,146$, 156, 168, 169, 189

Global x, xiii, xvi, 2, 4, 7, 9, 10, 18, 19, 26, 32, 35, 80, 81, 104, 109, $110,114,117,148,149$, 157-160, 163, 166, 167, 169, 174, 176, 178, 180-186, 193, 194, 204
Global companies $\mathrm{x}$

Globalisation vii, x, 15, 28, 32, 72, 79, 119, 192, 194, 196, 201-205

Google xvii, 204

Government policies $\quad$ xii, 71

Graduate students 149, 171,

Grants xiv, 34, 43, 139,

Great Dragon 4

Guangdong 37-40, 96, 155

Haier 3, 100, 112, 146, 182, 189

Haitian 156, 157

Hardware xi, 9, 23, 65, 153, 167, 174, 176, 180, 187

Heilongjiang Province 157

Hewlett Packard (HP) 21, 97, 160

High-tech industry xiv, 8-10, 14, 119-124, 130-132, 134-140, 142, 147,176

Hiring 167, 169

Hong Kong xvi, 17, 18, 32, 49, 68, 140, 146, 157, 163, 164, 174

Household xiv, 124-126, 128, 130, 131, 135, 137, 139, 141, 146, 157,180

Huawei $\quad 5,7,8,20,21,25,101,160$

Incentives $37,47,48,50,63,177,186$ Incubators $\mathrm{xv}, 152,161,183$

India $32,120,157,158,163,169,170$, 187,199

Indigenous technology 2

Individuals $\quad$ xii, 54-57, 125, 141, 191

Industrial concentration xii, 48, 61, 63,74

Industrial enterprises $\quad$ xii, 26, 54, 55, 77 Industrial structure $\quad 47,48,60,183$ Industry $\mathrm{x}$, xiii, xiv, xv, xvi, 1-5, 7-11, 13, 14, 16-21, 23, 25-30, 33, 48, 60-64, 70, 74, 84, 86-88, 91, 92, 95-98, 100, 101, 106, 107, 112, 114, 119-124, 128, 


\section{Index}

130-140, 142, 145-147, 154,

157-160, 165, 166, 168-171,

173-188, 191-193, 196, 201,

203, 205

Information xvi, 2, 3, 7, 9, 20, 24, 26, $36,45,55,57,61,74,75,82,86$, 87, 90, 91, 93-100, 103, 105, $111,112,145,148,150,152$, 157, 158-162, 164, 167, 168, 170, 171, 174-179, 183, 185, 186, 194-197, 202

Information and communications technology (ICT) 3, 4, 15, 85, 89, 174, 175, 184, 196

Infringements $16,18,20,70$

Innovation xi, xii, xvii, xviii, 3, 5,

6-8, 10, 20, 21, 23, 26-29, 33,

34, 36, 39-41, 47-49, 52-61, 63, 64, 67, 73-77, 79, 80, 84, 85, 91, 95, 108, 114, 131, 136, 139, 144, $145,147,148,170,177,178$, 183,202

Innovation activity $\quad$ xii, 41, 47-49, 77 Institute of Electrical and Electronics Engineers (IEEE) 81, 83, 107

Integration $2,28,35,48,93,153,166$, 176,178

Intel $10,21,101,119,160,178,180$, 189

Intellectual portfolios 32

Intellectual property $\mathrm{x}, 32,36,42,44$, 49, 50, 69, 81, 86, 88, 89, 93, 94, 103, 108, 169, 177, 185

Intellectual property rights $\mathrm{xi}, \mathrm{xiii}, 6$, 20, 24, 35-37, 43, 49, 50, 69-72, 77, 94, 98, 101, 111, 160

Internet $\mathrm{xv}, \mathrm{xvi}, 1,108,153,192,193$, 196, 197, 202, 203, 205

Inventions xii, 49, 50, 52, 53-56, 77

Investment $\mathrm{xii}, \mathrm{xv}, 1,4,5,15,16,20$, $21,29,31-33,44,45,47,50,60$, 61, 63, 67, 69-77, 81, 99, 111,
$121,123,125,130,131,137$, 144-146, 151, 152, 154, 155, 157, 159-164, 166, 171, 175, 180, 183-185, 192, 196, 209

Investors $15,50,63,73,75,77,131$, $155,164,183,185$

Iron and steel 3, 145, 166

Japan (Japanese) xiii, 1, 9, 18, 21, 26, 29, 32, 67, 68, 85, 89, 97, 100, $107,115,121,146,158-160$, 162, 164, 166, 169, 170, 173, $175,176,185,189$

Joint ventures (JV, JVs) 4, 57, 76, 91, 122

Knowledge 2, 23, 32, 33, 41-45, 61, 65, 75, 88, 143, 148, 184, 203, 205

Korea xiii, 18, 26, 27, 29, 32, 67, 100, 121, 146, 160, 163, 169, 170, 174,185

Labour ix, xv, xvi, 5, 7, 9, 14-18, 27, $126,136,145,158,180,182$

Labs xv, 96

Land xv, 63, 144, 150

Laptops 182

Large and medium-sized enterprises (LMEs) 57-60, 62, 137, 138

Laser 74, 95, 96, 176

Legal protection 37,48

Lenovo (Legend) 8, 19-21, 24, 28, 73, 100, 101, 112

Licences $\mathrm{xi}, \mathrm{xiii}, 155$

Licensing fees 32, 93

Life sciences $10,13,138,150$

Linux 4

Loans xiv, 124, 126-138, 142

Local content 70-72

Lower-end products 14

Low-tech industry 123 


\section{Index 217}

Machinery $\mathrm{xv}, 9,13,14,18,62,63$, 87, 146, 156-158, 189, 211

Managers xvi, 131, 152, 153, 155,

196, 202, 203, 205

Manufacturing $\quad \mathrm{x}, \mathrm{xi}, \mathrm{xv}, \mathrm{xvi}, \mathrm{xvii}, 4,5$,

7, 10, 13-15, 17, 20, 23, 27, 31, 61-66, 87, 92, 93, 96, 103, 113,

$117,121,123,131,138$,

145-147, 154-158, 163, 165,

167, 174-176, 178-180, 182-187,

189

Market forces xii, 76, 109, 112, 113, 144

Master's degrees (MA) 162, 166

Materials 9, 10, 12, 13, 62, 74, 75,

$147,154,164,165,176,177$,

188

Memory xvi, 10, 179

Microsoft xvii, 4, 10, 21, 94, 119, 160, 166,204

Ministry of Education 149-151, 170, 171

Ministry of Information Industry 86, $87,95,96,100,159,174,175$

Ministry of Science and

Technology 3, 10, 12, 21, 22,

24, 25, 34, 35, 38-40, 85, 94 , 104,177

Mitsubishi 18, 147

Mobile phones (see cell phones) xv, xvi, 90, 108, 155

Model xi, xii, xiv, 10, 24, 25, 44-46, $48,49,52,53,55,60,67,77$, 109, 120, 123-126, 128-132, $135,137,139,140,150,151$, 156, 160, 163, 165, 169, 176, $178,179,183,199$

Modification 24

Multinational companies (MNCs) xvi, 6, 7, 15, 18, 20, 21, 25, 26, 32, 64, 93, 101, 109, 144, 147, 160, 175, 181
National Development and Reform

Commission (NDRC) 87, 110, 166

Nationalism xiii, 2, 80, 81, 84, 109, 114, 116

NEC $18,97,160,166,184,189$

Netscape 119

Ningbo xv, 143-148, 150-156, 170

Nokia 19, 155, 160

Opto-electronics $\quad 10,12,138$

Originality 48

Outsourcing 5, 32, 157-159, 166, 170

Overhead 15

Panasonic 18, 97, 160, 167

Parks (High-tech parks) xv, 21, 75, 144, 148, 152, 157, 163

Patent law 35, 36, 42, 45, 47, 49, 50, $52,53,55$

Patent protection xii, 36, 45, 54, 76

Patents xi, xii, xiii, 6, 8, 18, 23-25, 31-33, 36-40, 44, 49, 50, 52-56, 59, 64, 69, 93, 98, 101,165

Pearl River 180,

Personal computers (PC) 1, 10, 19, 21, 29, 73, 182

Personnel 8, 21, 29, 57, 82, 166

Pharmaceutical industry 25

PhD degrees 148, 149, 162

Philips Co. Ltd. 18, 97, 164, 189

Pillar industries 74, 149

Pioneer Co Ltd 18, 148

Plan xiii, xiv, 84, 85, 90, 104, 114, 116, 149, 154, 158, 159, 162, 167, 171, 176, 177, 179, 183,187

Plan for Scientific Development xiii Pre-reform conditions xii

Price wars 16, 17, 20 


\section{Index}

Prices 51, 120-122, 138

Private 7, 48, 75, 145-147, 150, 151, 159, 161, 193, 194, 201

Processing 3, 13, 14, 16, 62, 63, 146, 153, 158, 159, 163, 182

Productivity xvii, 7, 36, 37, 40, 41, 48, $56,126,131,132$

Profits xiv, 16, 19, 40, 61, 108, 125, 126, 128, 130, 133, 135, 140, 197

Proprietary technologies xii, 32, 33, 37,44

Province xiv, 33, 38, 39, 45, 59, 146, $147,153,155,157,163,168$

Quality 5, 6, 27, 37, 56, 73, 76, 86, $95,115,131,132,140,154$, 159

Radio 6, 95, 96, 101, 102, 153

Refrigerators 146, 154, 180

Regional competition xii, 61

Regional differences 45,46

Regional protectionism xii, 61

Research and development

(R\&D) xi-xvi, 3-9, 21-23, 29,

31-34, 36, 38, 40, 41, 45, 47, 48,

50, 51, 53, 54, 56-64, 71, 72,

74-78, 80, 85, 86, 88, 92, 94, 95,

$112,113,123,139,156,157$,

159, 160, 164, 165, 167, 174,

184, 186, 187

Research institutes ix, x, xii, 20,30, $34,35,55,57,59,87,95,96$, 110, 148, 150, 168, 169

Restrictions 6, 70, 184, 185

Reverse-engineering 5

Radio frequency identification (RFID) 101-105, 107, 108

Risk xi, 10, 16, 21, 28, 61, 64, 72, 75, 76, 127-134, 139, 140, 176-179, 185,200

Royalties 18, 98, 102, 103, 108, 156
Sales 3, 5, 7-10, 18, 19, 21-23, 27, 58, 59, 61, 62, 67, 122, 138, 156-158, 160, 164, 174,

175,184

Samsung Co. Ltd. 25, 97, 147, 181, 185,189

Schumpeter 28, 60, 61, 63

Scientists $5,9,54,74,75,144,176$

Security 71, 81, 82, 102, 103, 105, 107, 111, 152, 153, 169, 180, 194

Semiconductors xvi, 3, 30, 164, 165, 167, 173-176, 178-181, 183, 185-187, 189

Shanghai xv, 38, 96, 97, 145, 150, $151,154,155,159,163$, 167, 180, 181, 187, 192-195, 203

Shenzhen 8, 104, 163, 169

Silicon Valley 29

Software $x v, 2,3,4,9,10,20,23,65$, $84,94,102,147,148,153$, 156-171, 177, 187

Sony $18,19,97,160,185,189$

State Economic and Trade Commission 3, 95, 99

State-owned enterprises (SOEs) xii, 3, 16, 21, 26, 42, 45, 49, 57, 60, 122,155

Statistics 5, 9-16, 21-25, 31, 34, 35, 37-40, 42-44, 65, 121, 122, 126, 138,174

Strategy xiii, 3-5, 7, 13, 20, 24, 26, 71-73, 80, 84-89, 104, 105, 109-111, 113-116, 170, 171, 185, 197, 201

Students xvi, 90, 149-152, 162, 165, $168,171,191$

Subsidisation xiv, 6, 75-77, 139, 146, 160, 168, 182

Suppliers $5,54,65,67,70,73,175$, 182, 188 
Supply xvii, 17, 23, 64, 77, 102, 103, 127, 130, 132, 134-136, 175, $178,180,188$

Survey $9,21,32,64,71,72,123$

Taiwan xvi, 18, 26, 32, 96, 100, 106, 146, 147, 174, 175, 178, 179, 181, 183, 184, 186, 189

Tariff $6,69,75$

Tax 63, 75, 122, 123, 144, 183

TCL (Corporation) 6, 8, 16, 17, 18, 26, 28, 72, 73, 77, 81, 87, 90, 96, 97, 99, 100, 101, 115, $116,155,156,164,180$, 189

Technology acquisition $\quad 63,73,74$

Technology exports $\quad 67,68$

Technology markets xi, xii, 33, 36, $38,39,42,44,45$

Technology transfer $4,6,14,27$, 36-38, 40, 41, 47, 50, 64, 65, 67, 69-74, 77, 78, 183

Telecommunications $\quad 5-7,9,10,12$, $13,24,62,63,90,91,112,113$, 138, 144, 167

Television (TV) 16, 17, 19, 20, 25, 95-97, 100, 106, 146, 153, 157, 167

Third Generation (3G) 2, 4-6, 25, 86, 90-92, 106, 112

Tianjin 39, 180, 181

Time Division-Synchronous Code

Division Multiple Access (TD-

SCDMA) 2, 6, 90-93, 108, 109, 112, 113, 115

Toshiba (Corporation) 6, 8, 18, 26, 28, 72, 73, 77, 81, 87, 90, 97, 99, 115, 116, 164, 180, 181, 185, 189

Trade deficit 13, 187

Trade surplus xiii, 13, 188

Trademarks 36, 69
Traditional industry xiv, 122-124, $131,132,137,139,140$

Training $71,136,144,150-153,160$, 161, 164, 166, 168, 187, 203

United States xiii, 1, 10, 15, 18, 49, 82, 90, 119, 121, 173, 175, 181, 184, 185, 189

University vii, 1-3, 20, 21, 27, 29, 30, $35,40,48,49,54,71,73,86,90$, 93, 94, 112, 140, 145, 147-154, 161, 162, 164, 168-171, 176, 178, 180, 184, 185, 187, 188

Utility model xi, xii, 24, 25, 49, 52, $53,55,60,77$

Value chain $10,16,18,79,178,203$

Value-added 16, 121, 122, 138, 145 , 183

Victor Company of Japan Ltd. (JVC) 18

Video 93-96

\section{Wages 15}

Weapons xiii, xvi, 10

Wired Authentication and Privacy Infrastructure (WAPI) 2, 81-84, 86, 89, 105, 107, 111, 113, 115, 116

Wireless 2, 4, 15, 81-83, 90, 92, 93, 112

Wireless Local Area Network (WLAN) 81

World Trade Organisation (WTO) xii, 12, 4, 6, 19, 32, 36, 43, 45, 48, 49, 53, 60, 69-72, 74, 76-80, 82, 84-86, 88, 105, 116, 183, 193

Zhejiang 37-40, 145, 146-154

Zhongxing (Co Ltd) 5, 7

Zone $\mathrm{xv}, 10,120,123,144,146$, 160-163, 177 